\title{
Approximating Optimal State Estimation
}

\author{
Brian F. Farrell and Petros J. Ioannou
}

\author{
Harvard University \\ Cambridge MA 02138, U.S.A \\ farrell@deas.harvard.edu,pji@cc.uoa.gr
}

\begin{abstract}
Minimizing forecast error requires accurately specifying the initial state from which the forecast is made by optimally using available observing resources to obtain the most accurate possible analysis. The Kalman filter accomplishes this for linear systems and experience shows that the extended Kalman filter also performs well in nonlinear systems. Unfortunately, the Kalman filter and the extended Kalman filter require computation of the time dependent error covariance matrix which presents a daunting computational burden. However, the dynamically relevant dimension of the forecast error system is generally far smaller than the full state dimension of the forecast model which suggests the use of reduced order error models to obtain near optimal state estimators. A method is described and illustrated for implementing a Kalman filter on a reduced order approximation of the forecast error system. This reduced order system is obtained by balanced truncation of the Hankel operator representation of the full error system and is used to construct a reduced order Kalman filter for the purpose of state identification in a time-dependent quasi-geostrophic storm track model. The accuracy of the state identification by the reduced order Kalman filter is assessed and comparison made to the state estimate obtained by the full Kalman filter and to the estimate obtained using an approximation to 4D-Var. The accuracy assessment is facilitated by formulating the state estimation methods as observer systems. Practical application of approximating to the reduced order Kalman filter making use of 4D-Var algorithms is examined.
\end{abstract}

\section{Introduction}

An important component of forecast error is error in the analysis of the initial state from which the forecast is made. Analysis error can be reduced by taking more observations, by taking more accurate observations, by taking observations at locations chosen to better constrain the forecast, and by extracting more information from the observations that are available. The last of these, obtaining the maximum amount of information from observations, is attractive because it makes existing observations more valuable and because, at least for linear systems, there is a solution to the problem of extracting the maximum information from a given set of observations: under appropriate assumptions the problem of extracting the maximum amount of information from a set of observations of a linear system in order to minimize the uncertainty in the state estimate is solved by the Kalman filter (KF) (Kalman, 1960; Ghil and Malanotte-Rizzoli, 1991; Wunch, 1996; Ide et al, 1997; Lermusiaux and Robinson, 1999). Moreover, application of the Kalman filter to the local tangent error equations of a nonlinear system provides a first order approximation to the optimal data assimilation method which is valid in the limit of sufficiently small errors. This nonlinear extension of the KF is referred to as the extended Kalman filter (EKF) (Ghil et al, 1981; Miller et al, 1994; Ide \& Ghil, 1997, Ghil, 1997).

Unfortunately, the Kalman filter and the extended Kalman filter require statistical description of the forecast error in the form of the error covariance and obtaining the required error covariance involves integrating a system with dimension equal to the square of the dimension of the forecast system. Direct integration of a system of such high dimension is not feasible. Attempts to circumvent this difficulty (see review of Ghil, 1997) have involved various approximations to the error covariance (Bishop et al, 2001; Tippett et al, 2000) 
and approximate integration methods (Evensen, 1994; Dee,1995; Fukumori and Malanotte-Rizzoli, 1995; Cohn and Todling, 1996; Verlaan and Heemink, 1997; Houtekamer and Mitchell, 1998).

While the formal dimension of the forecast error system obtained by linearizing the forecast model about a base trajectory is the same as that of the forecast system itself, there are reasons to believe that the effective dimension is far lower. The trajectory of the system state in a high dimensional dynamical system typically lies on a small dimensional subspace of the entire phase space. In chaotic systems all initial conditions approach this attractor which can be embedded in a space of dimension at most $2 d+1$, where $d$ is the attractor dimension (Takens, 1981). An estimate of the attractor dimension can be made from the number of positive Lyapunov exponents (the Kaplan-Yorke dimension; Kaplan \& Yorke, 1979) but anyway the attractor dimension is bounded above by the number of Lyapunov exponents associated with positive volume growth along the system trajectory in phase space (Illyashenko, 1983). While this is useful conceptually for bounding the dimension of the embedding space, identifying the subspace itself is more difficult in the case of nonlinear and time dependent systems. However, in the case of stochastically forced linear normal systems the analogous subspace to which the solution is primarily confined can be easily found by eigenanalysis of the covariance matrix of the system forced white in space and time. The resulting EOF spectrum typically falls off rapidly in physical models. The eigenvectors may be identified with the modes of the normal operator and the corresponding eigenvalues are the variance accounted for by the modes (North, 1984; Farrell and Ioannou, 1996, (henceforth FI96)). The fact that a restricted number of EOF's account for nearly all of the variance in normal systems shows that the effective dynamical dimension of these systems is small compared with the dimension of their phase space. This notion of quantifying the effective dimension of normal linear systems can be extended to bound the effective dimension of non-normal systems (Farrell and Ioannou, 2001a (henceforth FI01)).

In the case of the tangent linear forecast error system the spectrum of optimal perturbations of the error propagator over the forecast interval typically comprise a few hundred growing structures (Buizza and Palmer, 1995) and Lyapunov spectra for error growth have shown similar numbers of positive exponents (Palmer et al, 1998) which suggests from the above considerations that the effective dimension of the error system is $\mathrm{O}\left(10^{3}\right)$.

The problem of reducing the order of a linear dynamical system can be cast mathematically as that of finding a finite dimensional representation of the dynamical system so that the Eckart-Schmidt-Mirsky (ESM) theorem (Stewart and Sun, 1990) can be applied to obtain an approximate truncated system with quantifiable error. The ESM theorem states that the optimal $k$ order truncation of an $n$ dimensional matrix in the euclidean or Frobenius norm is the matrix formed by truncating the singular value decomposition of the matrix to its first $k$ singular vectors and singular values. A method for exploiting the ESM theorem to obtain a reduced order approximation to a dynamical system was developed in the context of controlling lumped parameter engineering systems and is called balanced truncation (Moore, 1981; Glover, 1984; Zhou and Doyle, 1998). Balanced truncation was applied to the set of ordinary differential equations approximating the partial differential equations governing perturbation growth in time independent atmospheric flows by FI01.

We first review the method of balanced truncation and apply it to a storm track model (cf Farrell and Ioannou, 2001b). We then review some salient aspects of optimal state estimation and discuss the structure of the gain matrix in the presence of model error and the asymptotic behavior of the assimilation error as the number of observations increases. We finally construct a reduced order Kalman filter based on balanced truncation and apply it to a time dependent Lyapunov unstable quasi-geostrophic model of a forecast tangent linear error system. 


\section{The storm track model}

\subsection{Formulating the model}

Consider an idealized model of the midlatitude storm track consisting of a Boussinesq atmosphere with constant stratification and constant shear in thermal wind balance on a $\beta$-plane channel with periodic boundary conditions in the zonal, $x$, direction; solid walls located at two latitudes in the meridional, $y$, direction and a solid lid at height $z=H$, simulating the tropopause. The observed zonal localization of a midlatitude storm track is simulated in the model by terminating the channel with a linear damping modelling the storm track exit region. The stability properties of such a storm track model are discussed in FI96.

Zonal and meridional lengths are nondimensionalized by $L=1200 \mathrm{~km}$; vertical scales by $H=f L / N=10 \mathrm{~km}$; velocity by $U_{0}=50 \mathrm{~m} / \mathrm{s}$; and time by $T=L / U_{0}$, so that a time unit is approximately $6.7 \mathrm{~h}$. The Brunt-Vaisala frequency is $N=10^{-2} s^{-1}$, and the Coriolis parameter is $f=10^{-4} s^{-1}$. The corresponding non-dimensional value of the planetary vorticity gradient is $\beta=0.46$.

The non-dimensional linearized equation which governs evolution of streamfunction perturbations is:

$$
\frac{\partial \nabla^{2} \psi}{\partial t}=-U(z) \nabla^{2} D \psi-\left(\beta-\frac{d^{2} U(z)}{d z^{2}}\right) D \psi-r(x) \nabla^{2} \psi
$$

in which the perturbation is assumed to be in the form $\psi(x, z, t) e^{i l y}$, where $l$ is the meridional wavenumber; $\nabla^{2} \psi$ is the perturbation potential vorticity, with $\nabla^{2} \equiv \partial^{2} / \partial x^{2}+\partial^{2} / \partial z^{2}-l^{2}$; and $D \equiv \partial / \partial x$. The perturbation potential vorticity damping rate $r(x)$ is taken to vary smoothly in the zonal direction with form:

$$
r(x)=\frac{\mu}{2}\left[2-\tanh \left(\frac{x-\pi / 4}{\delta}\right)+\tanh \left(\frac{x-7 \pi / 2}{\delta}\right)\right],
$$

in which parameters controlling the maximum damping rate and the width of the damping region have been chosen to be $\mu=5$ and $\delta=1.5$, respectively. The mean velocity profile is $U(z)=0.2+z$. The zonal extent of the re-entrant channel is $0<x<4 \pi$; latitudinal walls are located at $y=0$ and $y=1$, and the ground and tropopause boundaries are located at $z=0$ and $z=1$, respectively. In the following we consider perturbations with $l=1$. A cross section of the idealized storm track at a given latitude is shown in Fig 1. Conservation of potential temperature at the ground and tropopause provides the boundary conditions:

$$
\begin{gathered}
\frac{\partial^{2} \psi}{\partial t \partial z}=-U(0) D \frac{\partial \psi}{\partial z}+U^{\prime}(0) D \psi-r(x) \frac{\partial \psi}{\partial z}-\Gamma_{g}\left(D^{2}-l^{2}\right) \psi \text { at } z=0, \\
\frac{\partial^{2} \phi}{\partial t \partial z}=-U(1) D \frac{\partial \psi}{\partial z}+U^{\prime}(1) D \psi-r(x) \frac{\partial \psi}{\partial z} \text { at } z=1,
\end{gathered}
$$

where $U^{\prime}(0)$ and $U^{\prime}(1)$ denote the velocity shear at $z=0$ and $z=1$ respectively. The coefficient of Ekman damping $\Gamma_{g} \equiv \frac{N}{U_{0}} \sqrt{\frac{v}{2 f}}$ is given the value $\Gamma_{g}=0.0632$ corresponding to a vertical eddy momentum diffusion coefficient $v=20 \mathrm{~m}^{2} / \mathrm{s}$ in the boundary layer .

The waves evolve with nearly zero damping in the middle third of the channel (a length of $2 \pi L \approx 7500 \mathrm{Km}$ ) which models the core of the storm track. Because in this model absolute instabilities do not exist with everywhere westerly flow, the storm track is asymptotically stable for all meridional wavenumbers because all perturbations are eventually absorbed on entering the highly dissipative sponge (FI96).

Two scenarios are investigated. In the first a transiently growing disturbance excited near the western boundary of the storm track is modelled using the reduced order system, the purpose being to illustrate the accuracy of the reduced order model approximation of the autonomous dynamics. In the second, time dependence is added to 


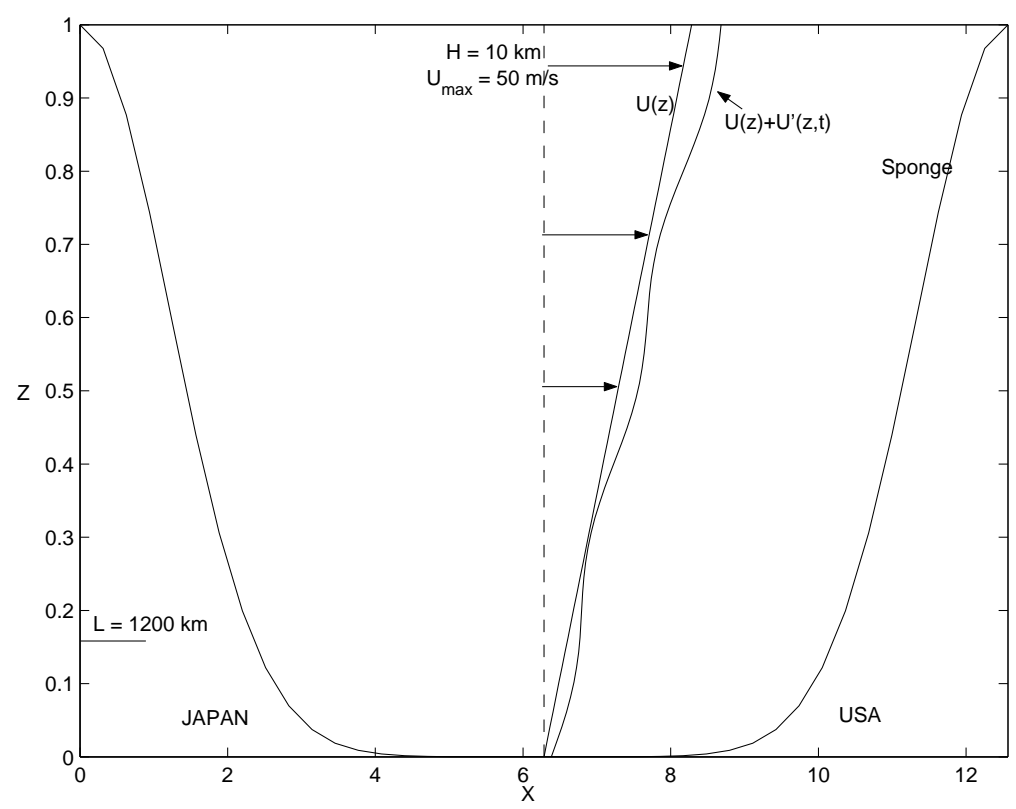

Figure 1: The cross section of the storm tack. Also shown are the sponge layers.

produce a Lyapunov unstable model of a tangent linear forecast error system, the time mean operator remaining stable, with the purpose of evaluating the accuracy of the Kalman filter obtained by the reduced order model in an unstable time dependent system. Such an unstable time dependent system provides an even more stringent test of the state estimator than does the time independent stable and unstable model error systems studied by Todling \& Ghil (1994), Ghil \& Todling (1996) and Cohn and Todling (1996).

The perturbation dynamics of the time mean storm track are governed by:

$$
\frac{d \psi}{d t}=\mathbf{A} \psi
$$

where:

$$
\mathbf{A}=\left(\nabla^{2}\right)^{-1}\left(-(0.2+z) D \nabla^{2}-\beta D-r(x) \nabla^{2}\right)
$$

in which the Helmholtz operator, $\nabla^{2}$, has been made invertible by incorporating the boundary conditions ${ }^{1}$.

The dynamical operator is approximated spectrally in the zonal direction and with finite differences in the vertical. With 40 zonal harmonics and 10 levels in the vertical the resulting dynamical system has $N=400$ degrees of freedom.

\subsection{Reducing the model order by balanced truncation}

Although this storm track model is of small enough dimension for direct numerical solution, we are interested in using it to explore the accuracy of approximate solutions obtained using reduced order models that could be implemented in far larger systems such as arise in numerical forecast.

Before proceeding with the order reduction we must first choose the norm that will be used to measure the accuracy of the approximation. The accuracy is measured by the norm of the euclidean length of the errors incurred in a chosen variable. This norm is the square root of the euclidean inner product in this variable. If another norm is selected to measure the accuracy of the approximation then the most direct method of accounting

\footnotetext{
${ }^{1}$ For waves with a constant meridional wavenumber $l$, the operator $\nabla^{2}$ is invertible even for homogeneous boundary conditions.
} 
for this choice is to transform the variable used to represent the state of the system so that the euclidean inner product in the transformed variable corresponds to the new norm. The reduced order approximate system resulting from balanced transformation will in general depend on the norm chosen. As discussed in FI01, optimal order reduction of dissipative stable normal systems is immediate: it is Galerkin projection of the dynamics onto the least damped modes. Difficulties in the reduction process arise when the system is non-normal in the variable corresponding to the chosen norm. Then Galerkin projection on the least damped modes is suboptimal and the reduction must proceed by including in the retained subspace the distinct subspaces of the preferred excitations and preferred responses of the system. Throughout this paper we have chosen streamfunction as the error variable, the rms of which is to be minimized in the construction of the model order reduction. However, we find that the results do not change qualitatively if the energy norm is chosen instead.

The preferred structures of response of the non-normal storm track system are revealed by stochastically forcing the system with spatially and temporally uncorrelated unitary forcing and calculating the eigenfunctions of the resulting mean covariance matrix $\mathbf{P}=\left\langle\psi \psi^{\dagger}\right\rangle$ (the brackets denote an ensemble average, and $\dagger$ the hermitian transpose of a vector or a matrix). The covariance matrix under such forcing is given by:

$$
\mathbf{P}=\int_{0}^{\infty} e^{\mathbf{A} t} e^{\mathbf{A}^{\dagger}} d t
$$

and this integral is readily calculated by solving the Lyapunov equation (FI96):

$$
\mathbf{A} \mathbf{P}+\mathbf{P} \mathbf{A}^{\dagger}=-\mathbf{I},
$$

which $\mathbf{P}$ satisfies, as can be easily verified. The hermitian and positive definite matrix $\mathbf{P}$ characterizes the response of the system and its orthogonal eigenvectors, ordered in decreasing magnitude of their eigenvalue, are the empirical orthogonal functions (EOF's) of the system under spatially and temporally uncorrelated forcing.

The preferred structures of excitation of the system are determined from the stochastic optimal matrix:

$$
\mathbf{Q}=\int_{0}^{\infty} e^{\mathbf{A}^{\dagger} t} e^{\mathbf{A}} d t
$$

the orthogonal eigenvectors of which when ordered in decreasing magnitude of their eigenvalue rank the forcing structures according to their effectiveness in producing the statistically maintained variance (for a deterministic interpretation of $\mathbf{Q}$ see FI01). The eigenvectors of $\mathbf{Q}$ are called the stochastic optimals (SO's) and because of the non-normality of the system are distinct from the EOF's. The stochastic optimal matrix $\mathbf{Q}$ satisfies the back Lyapunov equation:

$$
\mathbf{A}^{\dagger} \mathbf{Q}+\mathbf{Q} \mathbf{A}=-\mathbf{I}
$$

Lyapunov equations (8) and (10) have unique positive definite solutions $\mathbf{P}$ and $\mathbf{Q}$ if $\mathbf{A}$ is stable. The covariance matrix $\mathbf{P}$ and stochastic optimal matrix $\mathbf{Q}$ need to be determined or approximated in order to proceed with order reduction by balanced truncation.

A successful order reduction must accurately approximate the dynamics of the system which can be expressed as the mapping of all past (square integrable) forcings to all future responses of the system. This linear mapping of inputs to outputs is called the Hankel operator. Application of the ESM theorem to the Hankel operator provides the optimal low order truncation of the dynamics. Remarkably, because of the separation between past forcings and future responses in the Hankel operator representation of the dynamics this operator has finite rank equal to the order of the system; its singular values, denoted by $h$, are the square root of the eigenvalues of the product of the covariance and stochastic matrix, PQ. The balanced truncation transforms the internal coordinates of the system so that the transformed covariance matrix $\mathbf{P}$ and stochastic optimal matrix $\mathbf{Q}$ become identical and diagonal (while preserving the inner product of the physical variables). The dynamical system is then truncated in these transformed balanced coordinates. The balanced truncation retains a leading subset of 
empirical orthogonal functions (EOF's) and stochastic optimals (SO) of the dynamical system and preserves the norm. Balanced truncation preserves the stability of the full system and provides an approximation with known error bounds which is found in practice to be nearly optimal (Moore, 1981; Glover, 1984; FI01). The procedure used to implement the balanced truncation is now briefly reviewed.

Consider a general $k$ order truncation of the $N$ dimensional system (5):

$$
\frac{d \tilde{\psi}_{k}}{d t}=\mathbf{A}_{k} \tilde{\psi}_{k}
$$

where $\mathbf{A}_{k}$ is the reduced $k \times k$ dynamical matrix, with $k<N$, and $\tilde{\psi}_{k}$ the associated reduced order $k$-dimensional state vector which is related to the full state vector by the transformation $\tilde{\psi}=\mathbf{X} \tilde{\psi}_{k}$. Similarly, the reduced state vector $\tilde{\psi}_{k}$ is related to the full state vector by: $\tilde{\psi}_{k}=\mathbf{Y}^{\dagger} \tilde{\psi}$ (the dagger denotes the hermitian transpose of a matrix), which implies that $\mathbf{Y}^{\dagger} \mathbf{X}=\mathbf{I}_{k}$, where $\mathbf{I}_{k}$ is the $k$-order identity matrix. Matrices $\mathbf{Y}$ and $\mathbf{X}$ determine the transformation from the full system to the reduced system. The matrix $\mathbf{A}_{k}$, governing the dynamics in (11), is:

$$
\mathbf{A}_{k}=\mathbf{Y}^{\dagger} \mathbf{A} \mathbf{X}
$$

Details of the construction on the biorthogonal matrices $\mathbf{X}$ and $\mathbf{Y}$ are given in Farrell \& Ioannou (2001b).

A measure of the accuracy of the truncation is the maximum difference that can occur between the full system response, $\psi(t)$, and the reduced order system response, $\tilde{\psi}(t)$. This measure is the $H_{\infty}$ norm of the error system:

$$
\left\|\mathbf{A}-\mathbf{A}_{k}\right\|_{\infty}=\sup _{\omega}\|\mathbf{R}(\omega)-\tilde{\mathbf{R}}(\omega)\|_{2},
$$

in which the resolvent of the full system, $\mathbf{R}(\omega)$, is defined as $\mathbf{R}(\omega)=(i \omega \mathbf{I}-\mathbf{A})^{-1}$ and the resolvent of the full order projection of the reduced system is $\tilde{\mathbf{R}}(\omega)=\mathbf{X}\left(i \omega \mathbf{I}_{k}-\mathbf{A}_{k}\right)^{-1}$. It is to be recalled that the $L_{2}$ norm of a matrix, denoted as $\|\cdot\|_{2}$, is equal to its largest singular value.

Assuming the Hankel singular values have been ordered decreasing in magnitude, it can be shown that the error in the $H_{\infty}$ norm (13) of the approximation of the full system by any $k$ order system $\mathbf{A}_{k}$ satisfies the inequality:

$$
h_{k+1} \leq\left\|\mathbf{A}-\mathbf{A}_{k}\right\|_{\infty} \leq 2 \sum_{i=k+1}^{N} h_{i}
$$

where $h_{k+1}$ is the first neglected Hankel singular value (Zhou and Doyle, 1998). Although $h_{k+1}$ is only a lower bound on the error, we have found in examples that this lower bound is nearly attained.

\subsection{Applying balanced truncation to the mean storm track perturbation model}

In order to obtain a balanced truncation of the storm track model governed by operator (6) we first obtain the covariance matrix, $\mathbf{P}$, and the stochastic optimal matrix, $\mathbf{Q}$, by solving Lyapunov equations (8) and (10) respectively. The eigenfunction of $\mathbf{P}$ associated with the largest eigenvalue is the first EOF of the perturbation field, and the eigenfunction of $\mathbf{Q}$ associated with the largest eigenvalue is the first SO of the perturbation field. The structure of the first EOF, which accounts for $23 \%$ of the streamfunction perturbation variance, is concentrated in the exit region of the storm track as can be seen in Fig. 2 (top left panel). By contrast, the first $\mathrm{SO}$, which is responsible for generating $19.7 \%$ of the streamfunction perturbation variance, is concentrated at the entrance region of the storm track and is nearly orthogonal to the first EOF as can be also seen in Fig. 2 (bottom left panel). This near orthogonality between the EOF structures and SO structures remains even at order 30. Balanced truncation accomplishes an accurate representation of the dynamics by retaining both the structure of the dominant EOF's and of the SO's. It is clear from Fig. 2 that truncations based on projections 

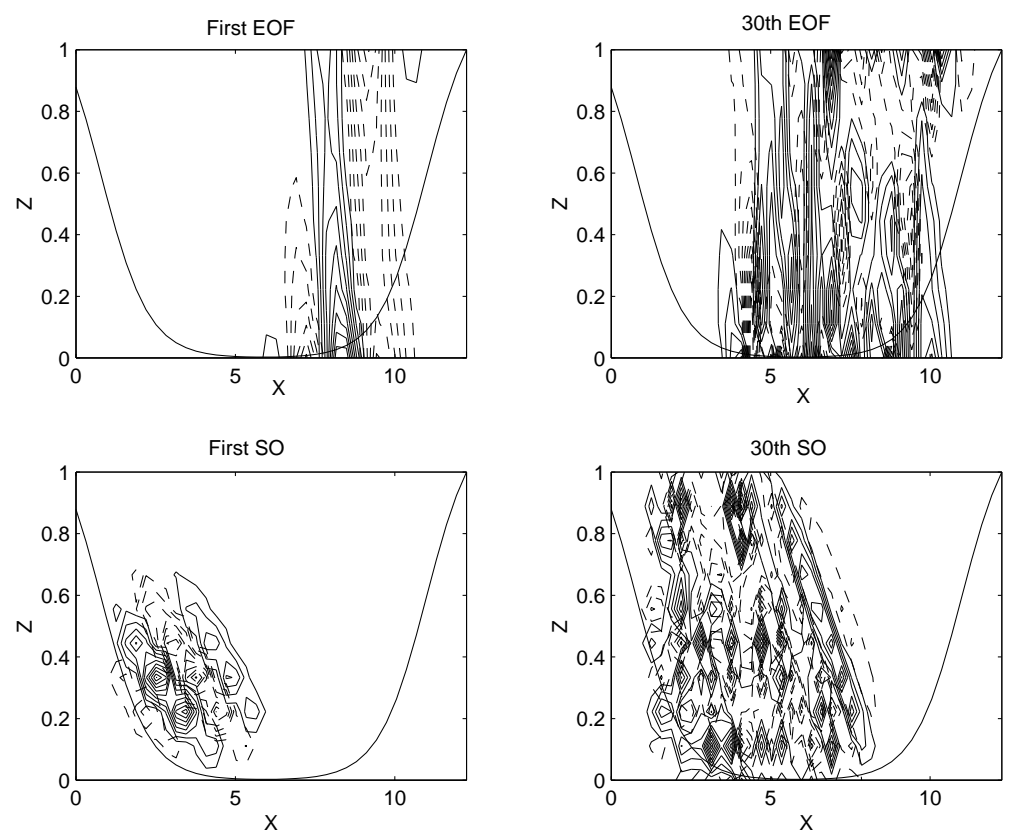

Figure 2: For the stable time mean storm track model. Top panels: The streamfunction of the first and the 30 th EOF. The first EOF accounts for $23 \%$ of the maintained variance, the 30th EOF accounts for $0.35 \%$ of the variance. Bottom panels: The structure of the streamfunction of the first and 30th Stochastic Optimal. The first SO is responsible for producing $19.7 \%$ of the maintained variance; the 30th SO is responsible for producing $0.48 \%$ of the maintained variance.

on the leading EOF's will be very suboptimal as the leading EOF's span well only the exit region of the storm track, leaving the dynamically important entry region of the storm track, where perturbations start growing, virtually without support in the span of the retained basis.

Although the error in the frequency response of a balanced truncation (cf (14)) is bounded above by twice the sum of the neglected Hankel singular values and below by the first neglected Hankel singular value, experience shows balanced truncation of tangent linear forecast error systems results in truncation errors close to the lower bound. The Hankel singular values and the eigenvalues of $\mathbf{P}$ and the $\mathbf{Q}$ for the storm track model are shown in Fig. 3.

Note that the decrease with mode number of the eigenvalues of $\mathbf{P}$ and of $\mathbf{Q}$ is more rapid than that of the Hankel singular values. But this more rapid decrease with mode number of the eigenvalues of $\mathbf{P}$ and $\mathbf{Q}$ does not indicate the order required for an accurate approximation; this is instead determined by the first neglected Hankel singular value which falls more slowly with mode number.

It is often assumed that a system can be well approximated by Galerkin projection onto a subspace of its EOF's; with the effectiveness of the truncation being judged from the magnitude of the eigenvalues of the neglected EOF's. While this is valid for normal systems, we see here that for non-normal systems the decrease with mode number of the eigenvalues of the covariance matrix is misleading and generally optimistic as an estimate of the order required for an accurate approximation.

A subset of the columns of $\mathbf{X}$ is retained in the balanced truncation. This non-orthogonal basis and its biorthogonal, the columns of $\mathbf{Y}$, are constructed so as to capture the structures supporting the dynamics most efficiently, simultaneously accounting for the preferred responses (EOF's) and the preferred excitations (SO's) of the dynamics. The first and the tenth structure retained in the dynamics (the first and the tenth column of $\mathbf{X}$ ) and their 


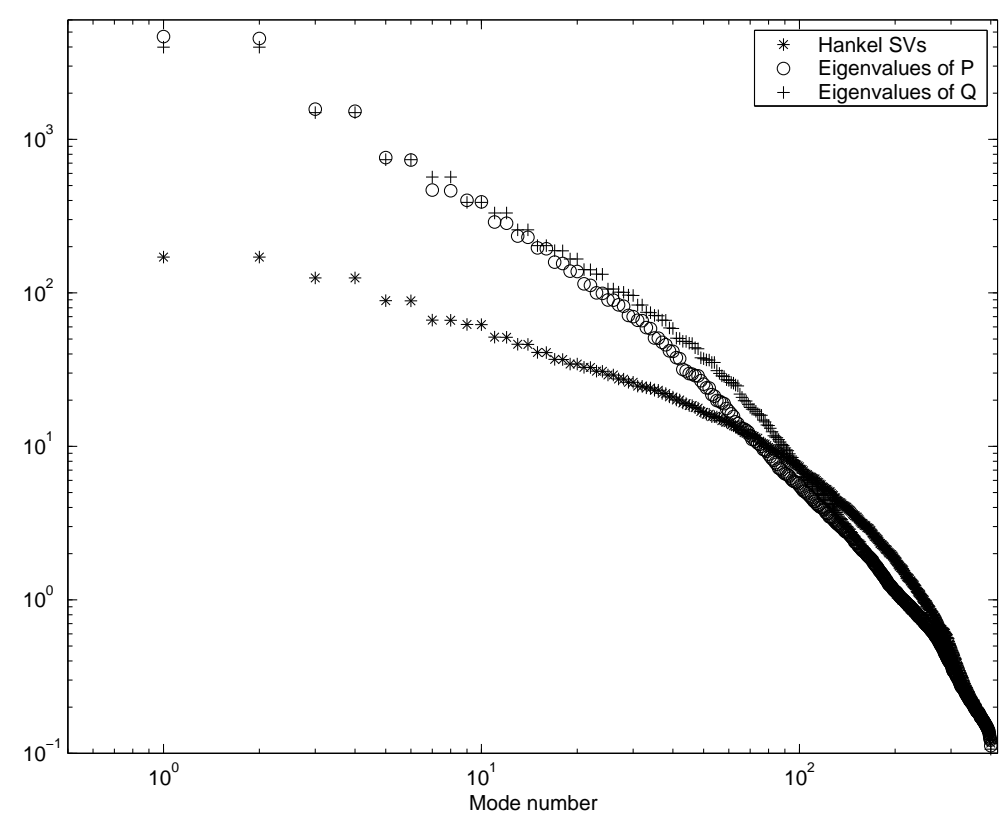

Figure 3: The Hankel singular values (stars) compared to the eigenvalues of the covariance matrix $\mathbf{P}$ (circles), and the eigenvalues of the stochastic optimal matrix $\mathbf{Q}$ (crosses). The Hankel singular values are the square roots of the eigenvalues of the product $\mathbf{P Q}$. Note that the EOF's (the eigenvalues of $\mathbf{P}$ ) and the SO's (the eigenvalues of $\mathbf{Q}$ ) fall much more rapidly with mode number than do the Hankel singular values.

biorthogonal structures (the first and tenth column of $\mathbf{Y}$ ) are shown in Fig. 4.

The storm track model and its reduced order approximate have very different eigenvalue spectra. The eigenvalue spectrum of the reduced order approximate is such that the frequency response of the approximate system is as close as possible to that of the original system, which is shown in Fig. 5. This results both from a decrease in the stability of the reduced system compared to that of the full system and from the increase in growth due to the non-normality in the reduced system.

The accuracy of the approximation is measured by the $H_{\infty}$ norm of the error dynamical system $\left\|\mathbf{A}-\mathbf{A}_{60}\right\|_{\infty}$, which, as discussed in the previous section, lies between the lower bound given by the first neglected Hankel singular value, $h_{61}=13.8$, and the upper bound: $2 \sum_{i=61}^{400} h_{i}=1.8 \times 10^{3}$. The largest singular value of the error system resolvent as a function of frequency is shown in Fig. 6, where it can be seen that $\left\|\mathbf{A}-\mathbf{A}_{60}\right\|_{\infty}=$ 28.5, which shows that the balanced truncation error in this example is only approximately twice its lower bound. The error is nearly white for the span of frequencies that correspond to the frequencies of the system eigenmodes. For comparison, the error incurred in an order 60 Galerkin projection of the dynamics onto the first 60 EOF's and the error incurred in an order 60 Galerkin projection onto the first 60 least damped modes, are also shown in Fig. 6. It can be seen that the EOF projection performs appreciably worse than the balanced truncation, while the modal truncation at this order is useless.

The optimal growth ${ }^{2}$ as a function of optimizing time attained by the full system and by the following: the order 60 balanced truncation; the order 60 system obtained by Galerkin projection on the first 60 EOF's; the order 60 system obtained by Galerkin projection on the first 60 SO's; and the order 60 system obtained by Galerkin projection on the first 60 least damped modes are all shown in Fig. 7. Note that the balanced truncation performs very well, reproducing the optimal growth nearly perfectly up to $t=5$, corresponding to

\footnotetext{
2 The optimal growth at time, $t$, is defined as the maximum perturbation growth that can occur over time $t$. For an autonomous system, governed by $\mathbf{A}$, the optimal growth at $t$ is given by the largest singular value of $e^{\mathbf{A} t}$ or by $\left\|e^{\mathbf{A} t}\right\|_{2}$.
} 

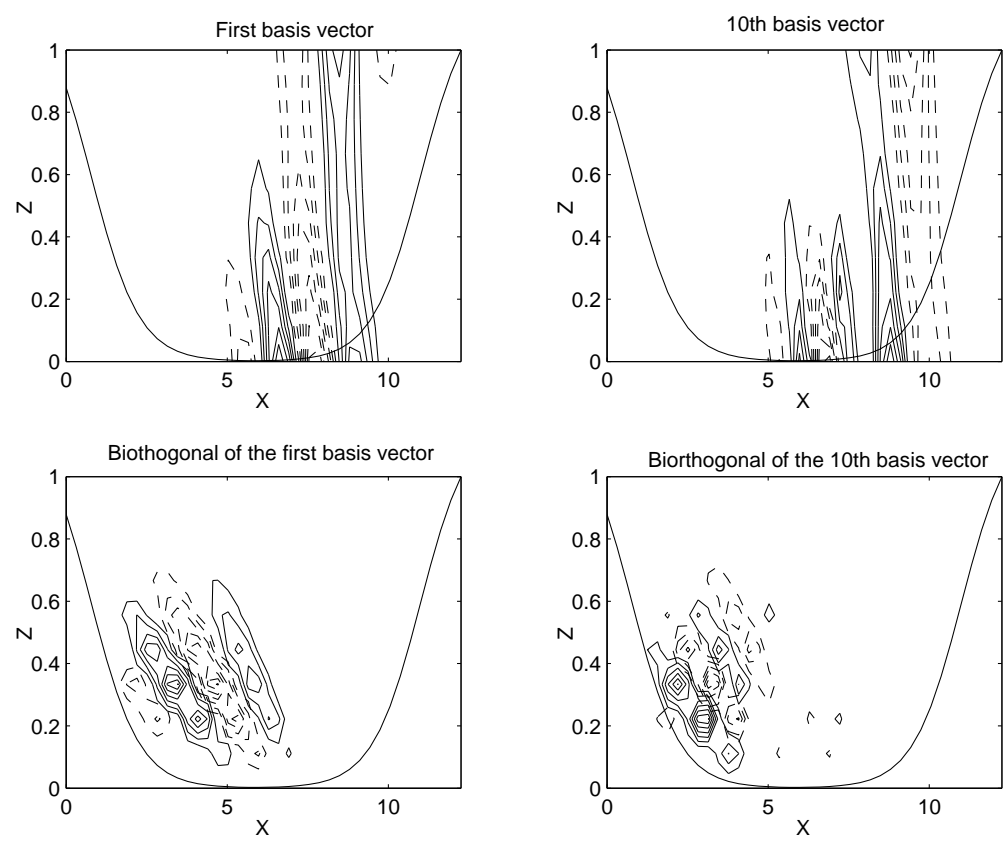

Figure 4: For the stable time mean storm track model. Top left panel: the streamfunction of the first basis vector of the expansion for the balanced truncation of the system. It is given by the first column of $\mathbf{X}$. Top right panel: the streamfunction of the tenth basis vector of the expansion for the balanced truncation of the system. It is given by the tenth column of $\mathbf{X}$. Bottom left panel: the streamfunction of the biorthogonal of the first basis vector. It is given by the first column of $\mathbf{Y}$. Bottom right panel: the streamfunction of the tenth basis vector. It is given by the tenth column of $\mathbf{Y}$.

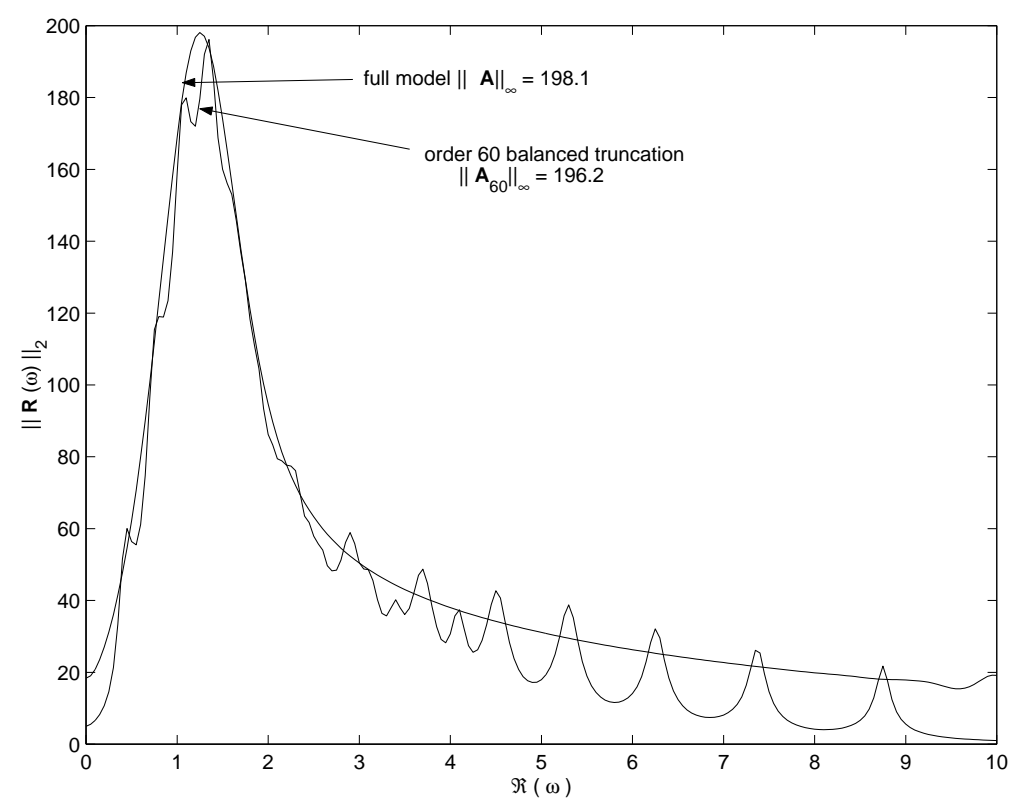

Figure 5: The maximum singular value of the resolvent $\mathbf{R}(\omega)=(i \omega I-\mathbf{A})^{-1}$ of the full system $\mathbf{A}$ as a function of frequency. The maximum of this curves as a function of $\omega$ is the $H_{\infty}$ norm of $\mathbf{A}$ which is found here to be 198.1. Also plotted is the maximum singular value of the resolvent associate with $\mathbf{A}_{60}$, which is the operator obtained from an order 60 balanced truncation of $\mathbf{A}$. The maximum of this curves is the $H_{\infty}$ norm of $\mathbf{A}_{60}$ which is found to be 196.2. 


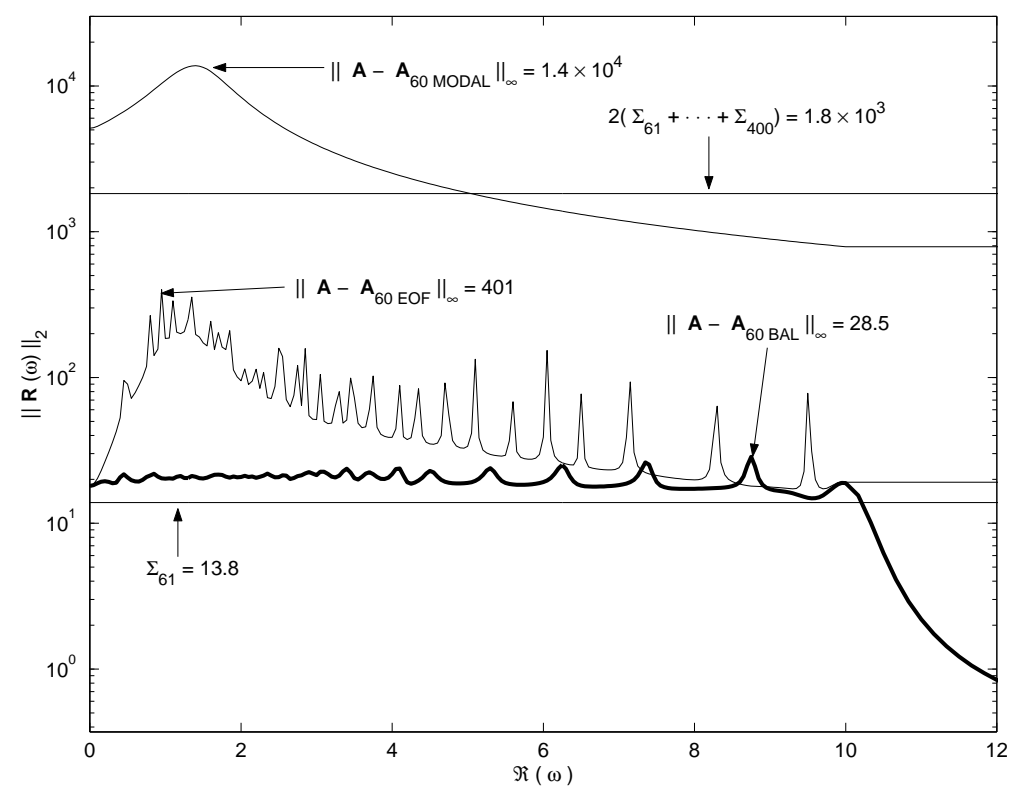

Figure 6: For the stable time mean storm track model: the maximum singular value of the error system $\mathbf{A}-$ $\mathbf{A}_{60}$ as a function of frequency. The system $\mathbf{A}_{60}$ is an order 60 approximation obtained from $\mathbf{A}$ by balanced truncation. The maximum of this curves is the $H_{\infty}$ error of the order 60 balanced truncation which is found here to be 28.5. Also indicated with a straight line is the theoretical minimum error of an order 60 truncation, which equals the first neglected Hankel singular value $\Sigma_{61}=13.8$. The balanced truncation is seen to be nearly optimal.

about 2 days. By comparison the EOF and SO truncations perform appreciably worse and the modal truncation gives even poorer results.

The structure of the initial perturbation that leads to greatest square streamfunction growth at $t=10$ in the full system, together with the resulting structure, is shown in Fig. 8; for comparison these structures as obtained by the truncated system are also shown. The structures are well captured by the order 60 reduced system.

We have demonstrated how to obtain balanced truncation of a stable time independent system but the method of balanced truncation can be extended to unstable systems (Sznaier et al, 2002) and to time dependent systems in which balancing is performed sequentially over finite time intervals (Van Dooren, 2000).

In forecast applications we seek an accurate reduction of the dynamics of the time dependent tangent linear operator calculated on the system trajectory over a limited time interval ( 24 or 48 hours). One choice is to balance on the time mean operator over this interval. Another choice is to balance on the time dependent version of the tangent linear operator over this or an extended interval about the assimilation time, obtaining approximation of the $\mathbf{P}$ and $\mathbf{Q}$ matrices on this interval. Both procedures have been tested using the time dependent version of our storm track model and found to produce accurate truncations. We examine below results obtained from a reduced order Kalman filter in which the truncation is made on the time dependent tangent linear operator over 48 hours centered on the assimilation time.

The time mean tangent linear operator (the mean being calculated over an interval) is generally asymptotically stable. This is because realistic states of the atmosphere support primarily instabilities with positive group velocities and do not support absolute instabilities (unstable modes with zero group velocity)(Farrell, 1982; Lin and Pierrehumbert, 1993; DelSole and Farrell, 1994). The asymptotic instability of the tangent linear system arises primarily from the continual instigation of transient growth which occurs in non periodic time dependent 


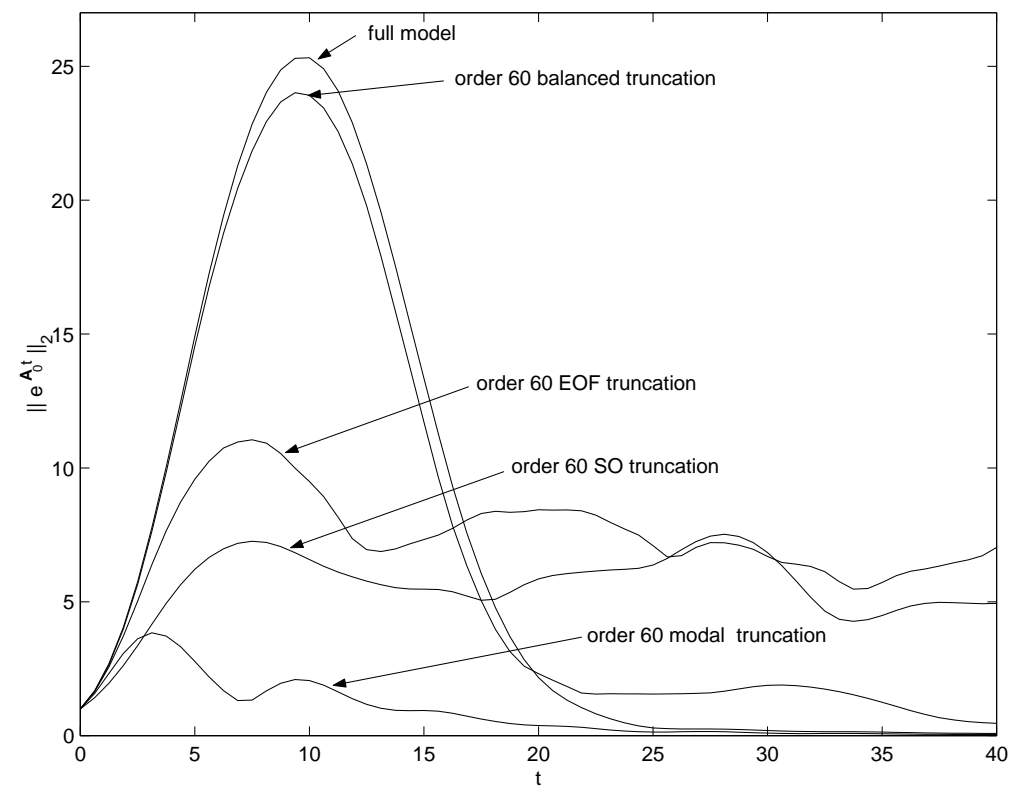

Figure 7: Optimal growth, $\left\|e^{\mathbf{A} t}\right\|$, as a function of time for the Eady model in a channel with sponge layers and meridional wavenumber $l=1$. Shown is the optimal growth for the full system with 400 degree of freedom and the optimal growth produced by an order 60 approximate system obtained by balanced truncation of the full system. Shown also for comparison is the optimal growth attained by the order 60 approximate system obtained by Galerkin projection on the first $60 \mathrm{EOF}$ 's, the first $60 \mathrm{SO}$ 's and the first 60 least damped modes.
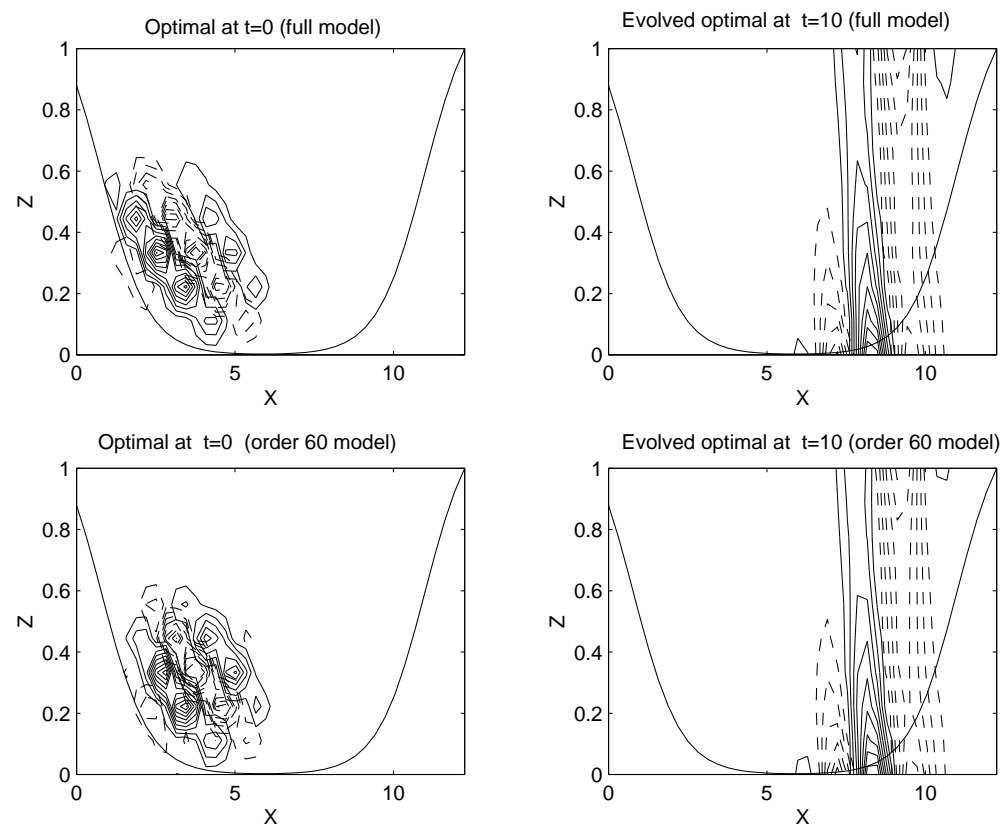

Figure 8: For the stable time mean storm track model. The structure of the streamfunction of the optimal perturbation that leads to the greatest energy growth at $t=10$ (left panels), and the evolved optimal streamfunction, which is the structure that these optimals evolve into at the optimizing time $t=10$ (right panels). The top panels are for the full system while the bottom panels are for the order 60 balanced truncation. 
systems in the same way that the Mathieu instability arises in time periodic systems. This mechanism is discussed in Farrell and Ioannou (1999) and has been verified in the context of a forecast system by Reynolds and Errico (1999) and Gelaro et al (2000). The stability of the mean operator allows balancing to be performed on a stable operator although the error system itself is nonautonomous and asymptotically unstable. However, it is not necessary to balance on the mean operator, and as remarked above comparable results can be obtained by balancing on the time dependent tangent linear operator over an appropriate interval; experiment suggests approximately 48 hours.

\section{Assimilation as an observer system}

Consider assimilating data taken from truth, $x_{t}$. The forecast error $e_{f}=x_{f}-x_{t}$ obeys the equation:

$$
\frac{d e_{f}}{d t}=\mathbf{A} e_{f}+\mathbf{Q}^{1 / 2} w_{m},
$$

in which $\mathbf{A}$ is the unstable tangent linear operator, $\mathbf{Q}$ is the model error covariance, and $w_{m}$ is assumed to be a vector of temporally uncorrelated noise processes.

Introduce $n$ observations, $y_{o b}$, defined in terms of truth $x_{t}$ as:

$$
y_{o b}=\mathbf{H} x_{t}+\mathbf{R}^{1 / 2} w_{o},
$$

where $\mathbf{R}$ is the observational error covariance and $w_{o}$ is an $n$ vector of white noise processes.

Assimilate these observations to obtain an analysis, $x_{a}$, with analysis error $e_{a}=x_{a}-x_{t}$ satisfying the Luenberger observer system:

$$
\begin{aligned}
\frac{d e_{a}}{d t} & =\mathbf{A} e_{a}+\mathbf{K}\left(y_{o b}-\mathbf{H} x_{a}\right)+\mathbf{Q}^{1 / 2} w_{m} \\
& =(\mathbf{A}-\mathbf{K H}) e_{a}+\mathbf{K R}^{1 / 2} w_{o}+\mathbf{Q}^{1 / 2} w_{m}
\end{aligned}
$$

The gain, $\mathbf{K}$, is chosen to minimize the analysis error variance trace $\left.\left(<\mathrm{e}_{\mathrm{a}} \mathrm{e}_{\mathrm{a}}^{\dagger}\right\rangle\right)$. Unlike the forecast error system, a Luenberger observer system is asymptotically stable. Any gain, $\mathbf{K}$, that stabilizes the tangent linear operator results in an observer with bounded error, this error being forced by a combination of model error $\mathbf{Q}$ and observational error $\mathbf{R}$ (cf 17). Good gains do not just stabilize the operator but simultaneously reduce the non-normality of the tangent linear operator so that the minimum of $\operatorname{trace}\left(<\mathrm{e}_{\mathrm{a}} \mathrm{e}_{\mathrm{a}}^{\dagger}>\right)$ is maintained by the combination of observational and model error.

Just as generalized stability of the tangent linear forecast system reveals the potential for forecast failures due to transient growth of initialization error or unresolved forcings distributed over the forecast interval, so also does generalized stability analysis of the observer system reveal how model error and initialization error contribute to analysis failures.

\subsection{The case of an optimal observer}

The gain $\mathbf{K}$ that minimizes the statistical steady analysis error variance trace $\left.\left(<\mathrm{e}_{\mathrm{a}} \mathrm{e}_{\mathrm{a}}^{\dagger}\right\rangle\right)$ is the Kalman gain. For simplicity of presentation we take as our example an operator $\mathbf{A}$ that is time independent and observations taken continuously in time. A stationary error system with continuous observations is chosen for heuristic reasons although in forecast systems the tangent linear operator is time dependent and observations are introduced at 
discrete intervals. However, the statistical properties of optimal state estimation are general and results are qualitatively similar across observer systems.

The asymptotic Kalman gain resulting from continual assimilation of observations with observation matrix $\mathbf{H}$ is:

$$
\mathbf{K}=\mathbf{P H}^{\dagger} \mathbf{R}^{-1}
$$

with $\mathbf{P}$ the stabilizing solution of the algebraic Ricatti equation:

$$
\mathbf{A P}+\mathbf{P A}^{\dagger}-\mathbf{P} \mathbf{H}^{\dagger} \mathbf{R}^{-1} \mathbf{H P}+\mathbf{Q}=0 .
$$

It is a property of the Kalman filter that the matrix $\mathbf{P}$ obtained as a solution of the algebraic Ricatti equation is also the asymptotic error covariance of the observer system (17).

\subsection{D-Var as an observer system}

4D-Var data assimilation with assimilation window $T$ can be viewed as a special case of an observer in which a climatological background error covariance $\mathbf{B}$ is advanced for $T$ units of time. In our autonomous model system the error covariance is advanced according to:

$$
\mathbf{P}=e^{\mathbf{A} T} \mathbf{B} e^{\mathbf{A}^{\dagger} T},
$$

from which we obtain the gain:

$$
\mathbf{K}_{4 D-V a r}=\mathbf{P H}^{\dagger}\left(\mathbf{H P H}^{\dagger}+\mathbf{R}\right)^{-1} .
$$

This gain produces a stabilized observer if enough observations are made.

The asymptotic error in the observer (17) is obtained by calculating the covariance, $\mathbf{P}$, that solves the equation:

$$
\left(\mathbf{A}-\mathbf{K}_{4 D-V a r} \mathbf{H}\right) \mathbf{P}+\mathbf{P}\left(\mathbf{A}-\mathbf{K}_{4 D-V a r} \mathbf{H}\right)^{\dagger}+\mathbf{K}_{4 D-V a r} \mathbf{R} \mathbf{K}_{4 D-V a r}^{\dagger}+\mathbf{Q}=0 .
$$

\section{Effect of the number of observations on the performance of the assimilation}

Consider convergence of the assimilated state to truth as more observations are taken in the presence of model error. To fix ideas assume that repeated independent observations are made at each of the grid points of our model.

If the state of the assimilation system has dimension $N$ and $n$ observations are taken at each grid point the observation matrix for these $n$ observations, $\mathbf{H}_{n}$, is an $n N \times N$ matrix:

$$
\mathbf{H}_{n}=\mathbf{I}_{N} \bigotimes e
$$

where $\mathbf{I}_{N}$ is the identity $N^{2}$ dimensional matrix, $\otimes$ denotes the Kronecker product and $e$ is the unit column $e=[1, \cdots, 1]^{T}$ of dimension $n$.

Consider an observation error covariance matrix $\mathbf{R}=r \mathbf{I}_{N} \otimes \mathbf{I}_{n}$, where $\mathbf{I}_{n}$ is the $n^{2}$ dimensional identity matrix and let $\mathbf{K}_{n}$ be the Kalman gain that results from these $n$ observations. The Kalman gain is:

$$
\mathbf{K}_{n}=\mathbf{P}_{n} \mathbf{H}_{n}^{\dagger} \mathbf{R}^{-1}=\frac{1}{r} \mathbf{P}_{n}\left(\mathbf{I}_{N} \bigotimes e^{\dagger}\right)
$$

with $\mathbf{P}_{n}$ the stabilizing solution of the algebraic Ricatti equation:

$$
\mathbf{A} \mathbf{P}_{n}+\mathbf{P}_{n} \mathbf{A}^{\dagger}-\mathbf{P}_{n} \mathbf{H}_{n}^{\dagger} \mathbf{R}^{-1} \mathbf{H}_{n} \mathbf{P}_{n}+\mathbf{Q}=0
$$


where $\mathbf{Q}$ is the model error covariance. On substitution of the specific expressions above for the observation matrix $\mathbf{H}_{n}$ and the observational error covariance matrix $\mathbf{R},(25)$ assumes the simplified form:

$$
\mathbf{A} \mathbf{P}_{n}+\mathbf{P}_{n} \mathbf{A}^{\dagger}-\frac{n}{r} \mathbf{P}_{n}^{2}+\mathbf{Q}=0,
$$

from which we conclude that the analysis error in the observer system resulting from assimilation of $n$ observations at each grid point with each observation having observational error variance $r$ is equal to the analysis error that results from observing the same system with a single isolated observation with observational error variance $r / n$. It remains to determine how the error covariance $P_{n}$ scales with $n$.

In the absence of model error $(\mathbf{Q}=0)$ the answer is immediate:

$$
\mathbf{P}_{n}=\frac{\mathbf{P}}{n},
$$

where $\mathbf{P}$ is the assimilation error covariance associated with a single observation which satisfies the algebraic Ricatti equation:

$$
\mathbf{A P}+\mathbf{P A}^{\dagger}-\frac{1}{r} \mathbf{P}^{2}=0 .
$$

So in the absence of model error the assimilation square error tends to zero as more observations are taken at the expected rate of $n^{-1}$.

Consider now the case in which model error exists. In that case we may expand $P_{n}$ in an asymptotic series:

$$
\mathbf{P}_{n}=\frac{p_{o}}{\sqrt{n}}+\frac{p_{1}}{n}+\cdots .
$$

The leading term in this expansion is given by:

$$
p_{o}=\sqrt{r} \mathbf{Q}^{1 / 2},
$$

and consequently the asymptotic error covariance in the presence of model error has the leading behavior:

$$
\mathbf{P}_{n}=\sqrt{\frac{r}{n}} \mathbf{Q}^{1 / 2} .
$$

We conclude that in the presence of model error the assimilation square error of the Kalman filter tends to zero in our example as more observations are taken at rate $n^{-1 / 2}$.

It is instructive to compare this to the behavior of analysis error in a 4D-Var data assimilation as the number of observations increases. In the absence of model error the 4D-Var analysis square error also tends to zero at rate $n^{-1}$, but in the presence of model error if the background covariance $\mathbf{B}$ is not rescaled as more observations are taken the analysis error asymptotes to a non zero constant value.

In order to understand this behavior consider the asymptotic error as $n \rightarrow \infty$ in the unstable stochastically forced scalar system with growth rate $a$ :

$$
\frac{d e}{d t}=a e+q^{1 / 2} w .
$$

The associated algebraic Ricatti equation is:

$$
2 a p_{n}-\frac{n}{r} p_{n}^{2}+q=0
$$

with stabilizing solution:

$$
p_{n}=a \frac{r}{n}+\sqrt{a^{2}\left(\frac{r}{n}\right)^{2}+q \frac{r}{n}}
$$


FARRELl \& IOANNOU.: APPROXIMATING OPTIMAL STATE ESTIMATION

Time invariant scalar model

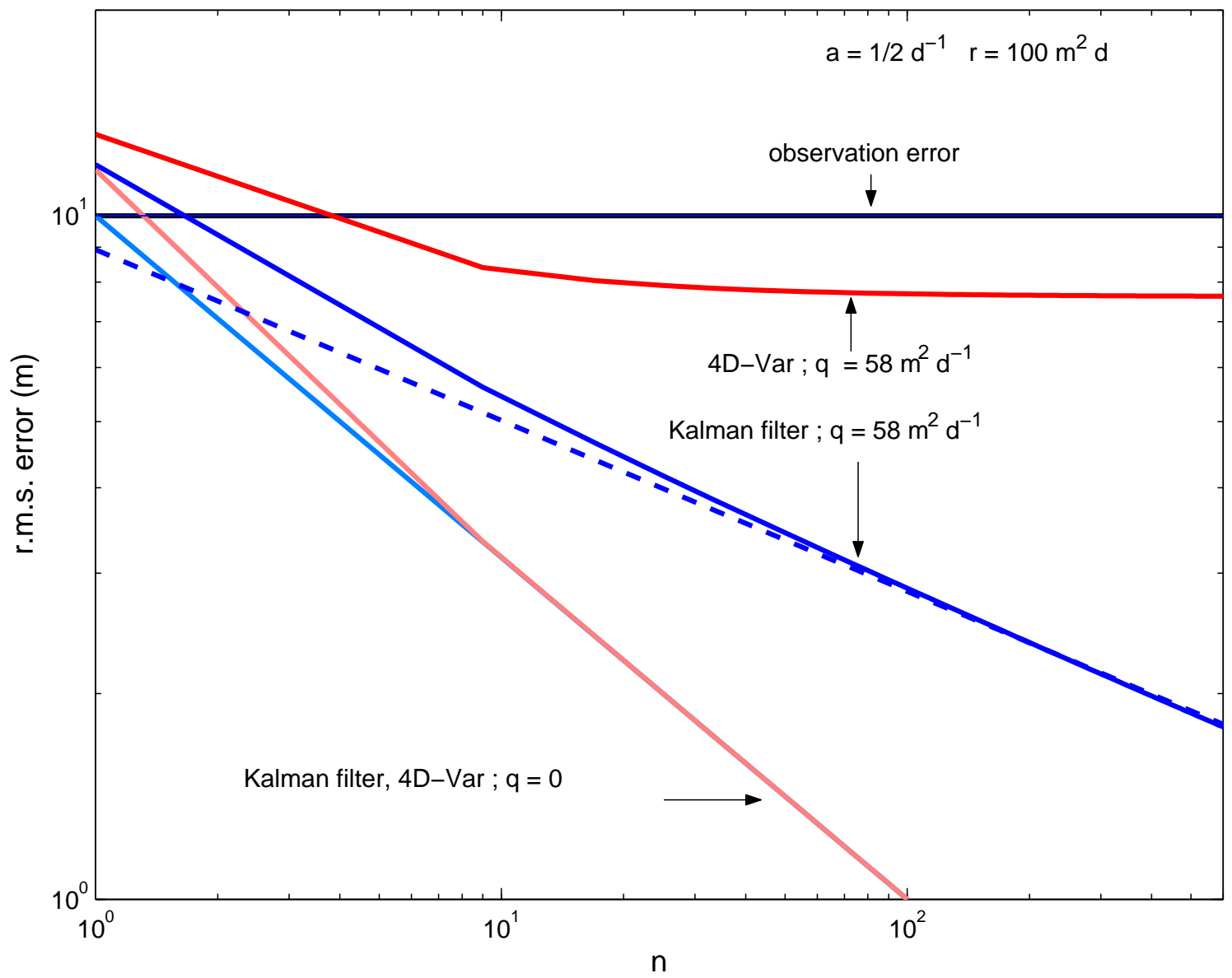

Figure 9: Error in the scalar optimal observer system and a scalar system with an equivalent 4D-Var observer as a function of the number of observations. The gain in the optimal observer is the asymptotic Kalman gain. The growth rate is $a=1 / 2 d^{-1}$, the observational error is $10 \mathrm{~m}$. The model error variance is $q=58 \mathrm{~m}^{2} d^{-1}$ resulting in a model induced error of $10 \mathrm{~m}$ after a day. With $q=0$ the error in both the observer system with the Kalman filter and the $4 D$-Var falls as $n^{-1 / 2}$. With $q \neq 0$ the error in the $4 D$-Var observer asymptotes to a constant value while in the observer with the Kalman filter falls as $n^{-1 / 4}$. 
This stabilizing solution is also the error in the observer system after assimilation of $n$ observations. Note that in the absence of model error and for all $n$ :

$$
p_{n}=\frac{2 a r}{n} \quad \text { if } q=0
$$

and that the Kalman gain is

$$
\mathbf{K}_{n}=\frac{2 a}{n}[1,1, \cdots, 1,1]
$$

and that the weight given each in the assimilation is:

$$
\mathbf{K}_{n} \mathbf{H}_{n}=2 a
$$

indicating that the weight given to observations is proportional to the error growth rate and is independent of the number of observations.

With model error and as $n \rightarrow \infty$ :

$$
p_{n} \approx \sqrt{\frac{q r}{n}} \text { if } q \neq 0
$$

and the Kalman gain is:

$$
\mathbf{K}_{n} \approx \sqrt{\frac{q r}{n}}[1,1, \cdots, 1,1],
$$

so that the weight given to observations is:

$$
\mathbf{K}_{n} \mathbf{H}_{n}=\sqrt{n q r},
$$

independent of error growth rate and indicating that as the number of observations tends to infinity in the presence of model error the model is increasingly discounted and the observations accepted. A comparison of the error as a function of the number of observation in the scalar system is shown in Fig. 9.

Regardless of the model error, the error in the optimal observer vanishes if enough observations are assimilated a result that holds in higher dimensions, as we have seen.

\section{Approach of 4D-Var to the Kalman filter as the assimilation interval in- creases}

In the absence of model error 4D-Var is equivalent to the extended Kalman filter if the assimilation window is extended to infinity. Present implementations of 4D-Var employ assimilation windows of 12 hours and it may appear that these implementations must be suboptimal and that the assimilation could be improved by lengthening the assimilation window.

Consider the asymptotic gain arising from a single observation in the time independent storm track model with and without model error. The asymptotic gain is shown in Fig. 10 (top panel). It is evident that in the presence of model error the gain is not localized: the gain identifies the unstable structures of the forecast model and provides loadings designed to destroy these structures which have the character of a global mode. As shown in Fig. 10 (bottom panel) in the presence of model error the gain becomes localized to the neighborhood of the observation because the model error that is distributed in the system produces incoherent responses far from the observation location that cancel when the ensemble average response of the system is taken so that the gain in the presence of model error is localized.

Because 4D-Var calculates the gains without model error the gain associated with a 4D-Var assimilation as the assimilation window is increased extends into the far field. This evolution of the gain associated with an initial 

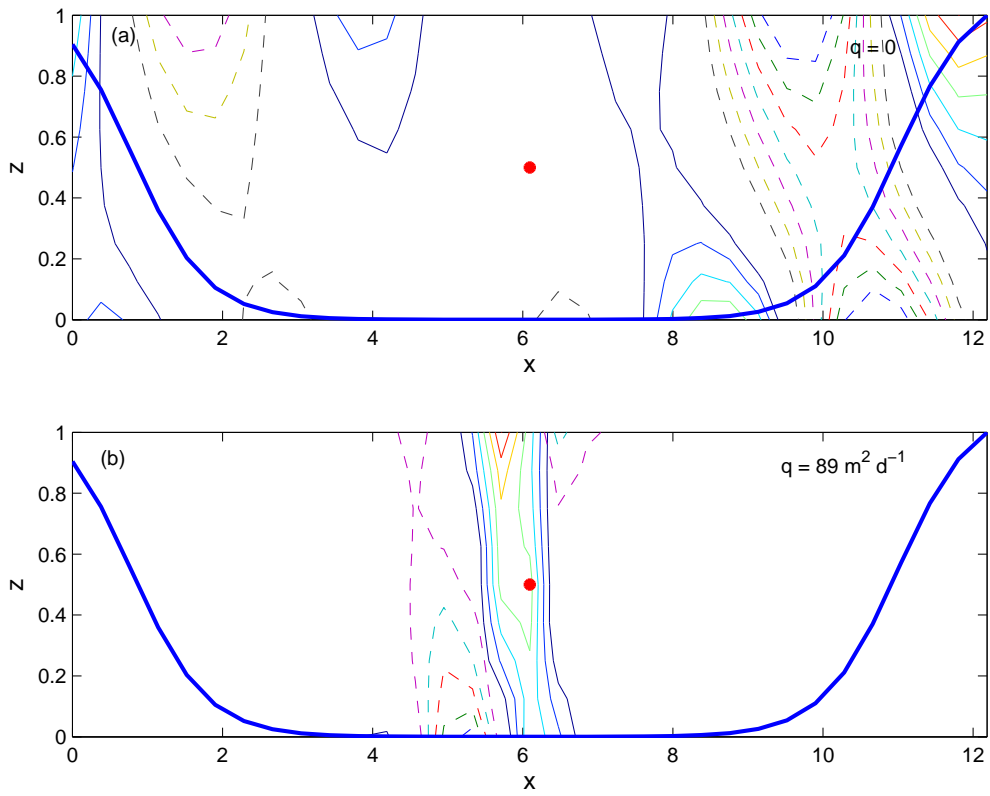

Figure 10: The asymptotic Kalman gain for observation at the center of the channel in the storm track model. Top panel the gain for the case of no model error. Bottom panel the gain for the case with model error. The model error $q$ produces an r.m.s. model error of $5 \mathrm{~m}$ in a day. The r.m.s. observational error is $10 \mathrm{~m}$. The asymptotic Kalman gain has been calculated for the time mean flow. Note that the model error leads to localization of the gain in the neighborhood of the observations.
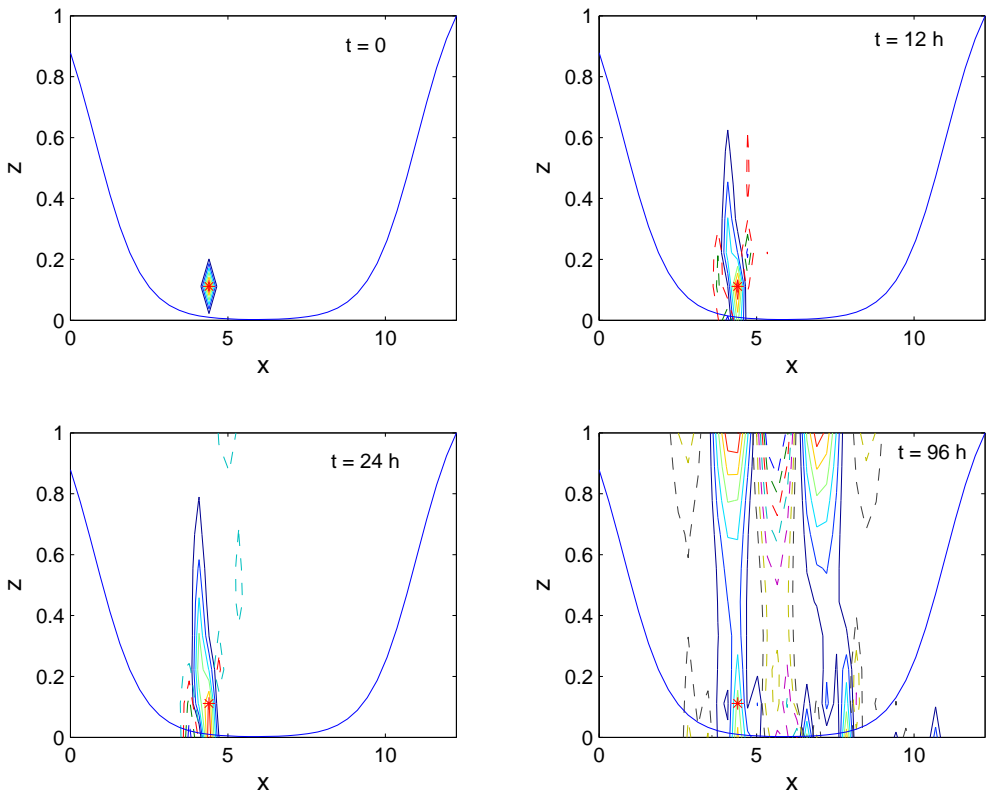

Figure 11: Evolution of the gain associated with the observation marked with a star in 4D-Var as a function of the assimilation interval in the unstable time mean storm track error model. The background $\mathbf{B}$ matrix is the identity. As the assimilation interval increases $4 D$-Var gains extend into the far field. 


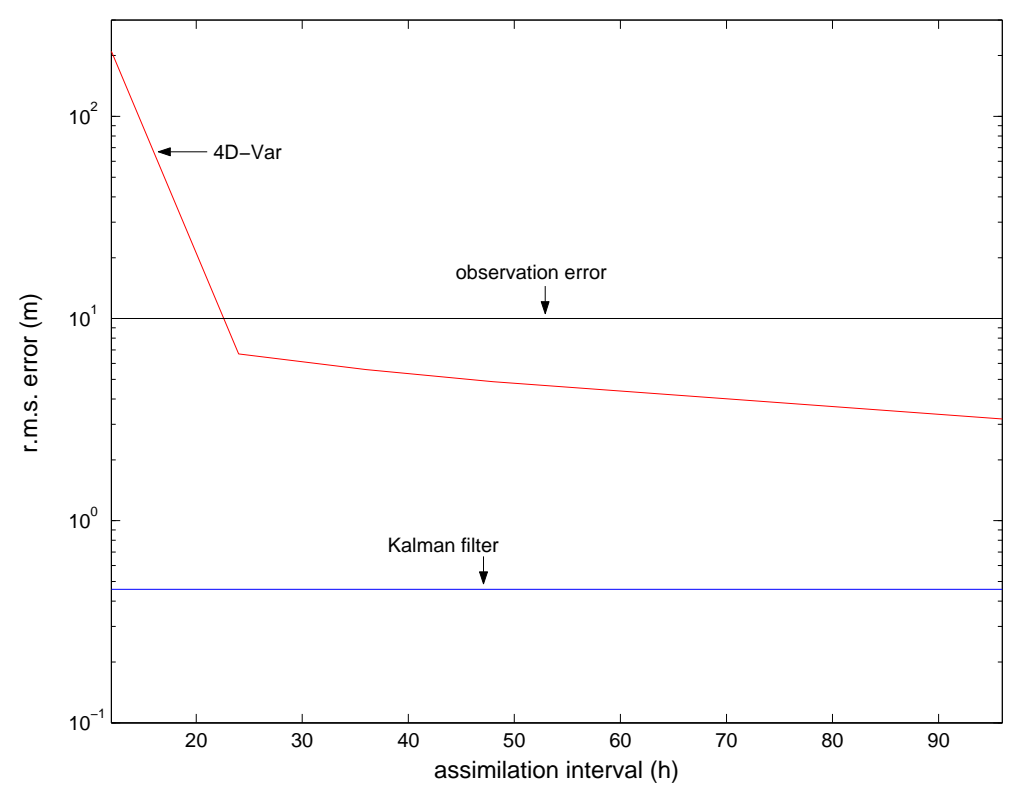

Figure 12: Error in 4D-Var assimilations in the time dependent storm track model with no model error as a function of assimilation interval. Also shown is the error obtained with sequential application of a Kalman filter. 16 observations are assimilated with r.m.s. observational error of $10 \mathrm{~m}$. As the assimilation interval tends to infinity the 4D-Var error approaches that of the Kalman filter.

climatological background $\mathbf{B}$ in a 4D-Var assimilation is shown in Fig. 11. With time the climatological gain associated with the background error covariance assumes a global structure.

In the absence of model error the gain as the assimilation interval increases approaches the structure of the gain of the Kalman filter and the analysis error of 4D-Var asymptotes to the analysis error obtained by a Kalman filter. The convergence of 4D-Var assimilation error to that of the Kalman filter is shown for the time dependent version of the storm track model in Fig. 12.

However, the perfect model assumption is physically unrealistic, and the 4D-Var assimilation scheme produces gains that have global structure as the assimilation window is increased. We find in our model storm track that 4D-Var performs best with an assimilation window that is large enough to allow the gain to be affected by the flow but short enough so that far-field loadings do not have time to form. An example of 4D-Var analysis error as a function of the assimilation interval is shown in Fig. 13. In this example the optimal assimilation interval is 36 hours.

We conclude that neglect of model error in the formulation of 4D-Var makes 4D-Var operate best for rather short assimilation intervals. Model error must be introduced to make 4D-Var an optimal observer. In the sequel we propose a method for introducing model error into 4D-Var.

\section{Reduced order error covariance estimate}

We now formulate the observer system in which the error covariance is advanced in the truncated space to obtain a reduced order Kalman gain. The resulting observer system in reduced coordinates is:

$$
\frac{d e_{k}}{d t}=\left(\mathbf{A}_{k}-\mathbf{K}_{k} \mathbf{H}_{k}\right) e_{k}+\mathbf{K}_{k} \mathbf{R}_{k}^{1 / 2} w_{o}-\mathbf{Q}_{k}^{1 / 2} w_{m} .
$$




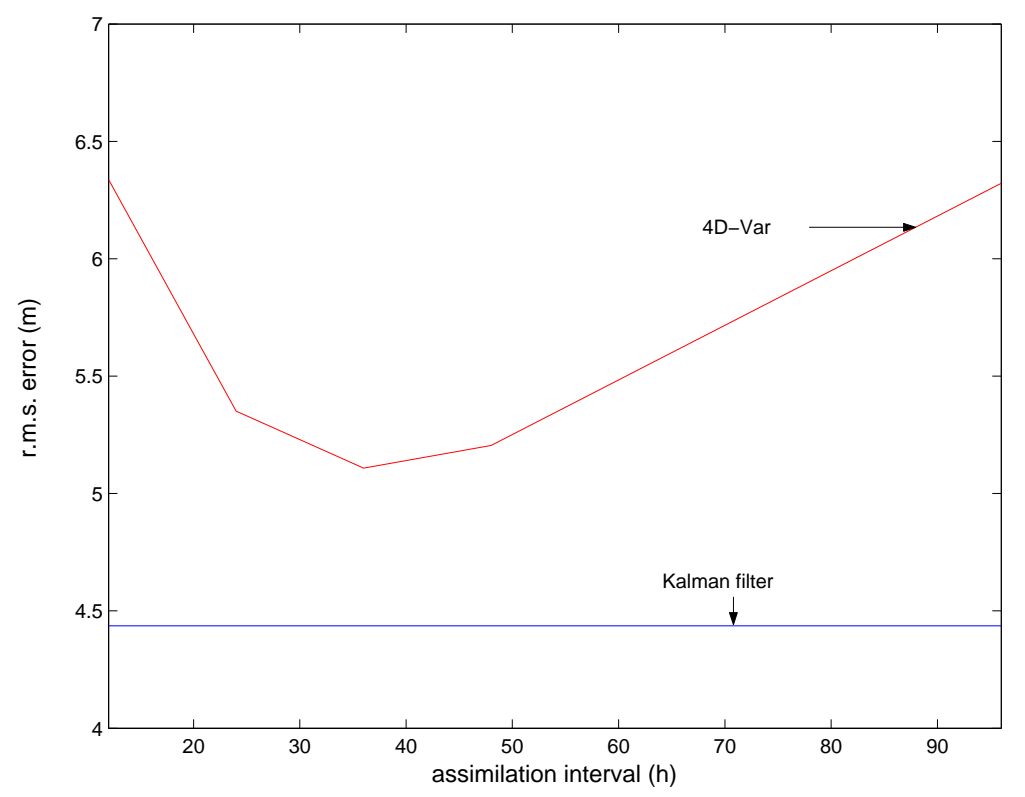

Figure 13: R.m.s. error in 4D-Var assimilations in the time dependent storm track model with model error as a function of assimilation interval. The best $4 D$-Var performance is achieved in this example for assimilation over the interval 36 h. Also shown is the error obtained with the Kalman filter. 40 observations are assimilated with r.m.s. observational error of $10 \mathrm{~m}$; the model error variance is $q=12 \mathrm{~m}^{2} \mathrm{~d}^{-1}$, so that a model error of $5 \mathrm{~m}$ accumulates in one day.

where the reduced analysis is $e_{k}=\mathbf{Y}^{\dagger} e_{a}$ for $k<<N$ and the reduced $k \times k$ operator is:

$$
\mathbf{A}_{k}=\mathbf{Y}^{\dagger} \mathbf{A X} .
$$

The $n$ observations, $y_{o b}$, are assimilated in the reduced space according to:

$$
y_{o b}=\mathbf{H}_{k} x_{k}+\mathbf{R}^{1 / 2} w_{o},
$$

where the reduced order observation matrix is:

$$
\mathbf{H}_{k}=\mathbf{H} \mathbf{X} \text {. }
$$

The error system in the reduced space is used to obtain the Kalman gain $\mathbf{K}_{k}$ and to propagate the error covariance,

$$
\mathbf{P}_{k}=<e_{k} e_{k}^{T}>.
$$

The error covariance of the full system is then approximated from that of the reduced covariance $\mathbf{P}_{k}$ by:

$$
\mathbf{P}=\mathbf{X P}_{k} \mathbf{X}^{\dagger} .
$$

This error covariance is used in our 4D-Var model. By introducing this covariance in 4D-Var we evolve the error covariance and simultaneously also introduce model error. Introduction of this reduced order covariance in 4D-Var makes the 12 hour 4D-Var perform nearly optimally. Analysis of the performance of this filter is shown in Fig. 14. Using the reduced order covariance obtained without model error leads to degradation of the 4D-Var assimilation due to unrealistic far field loadings in the gains. 

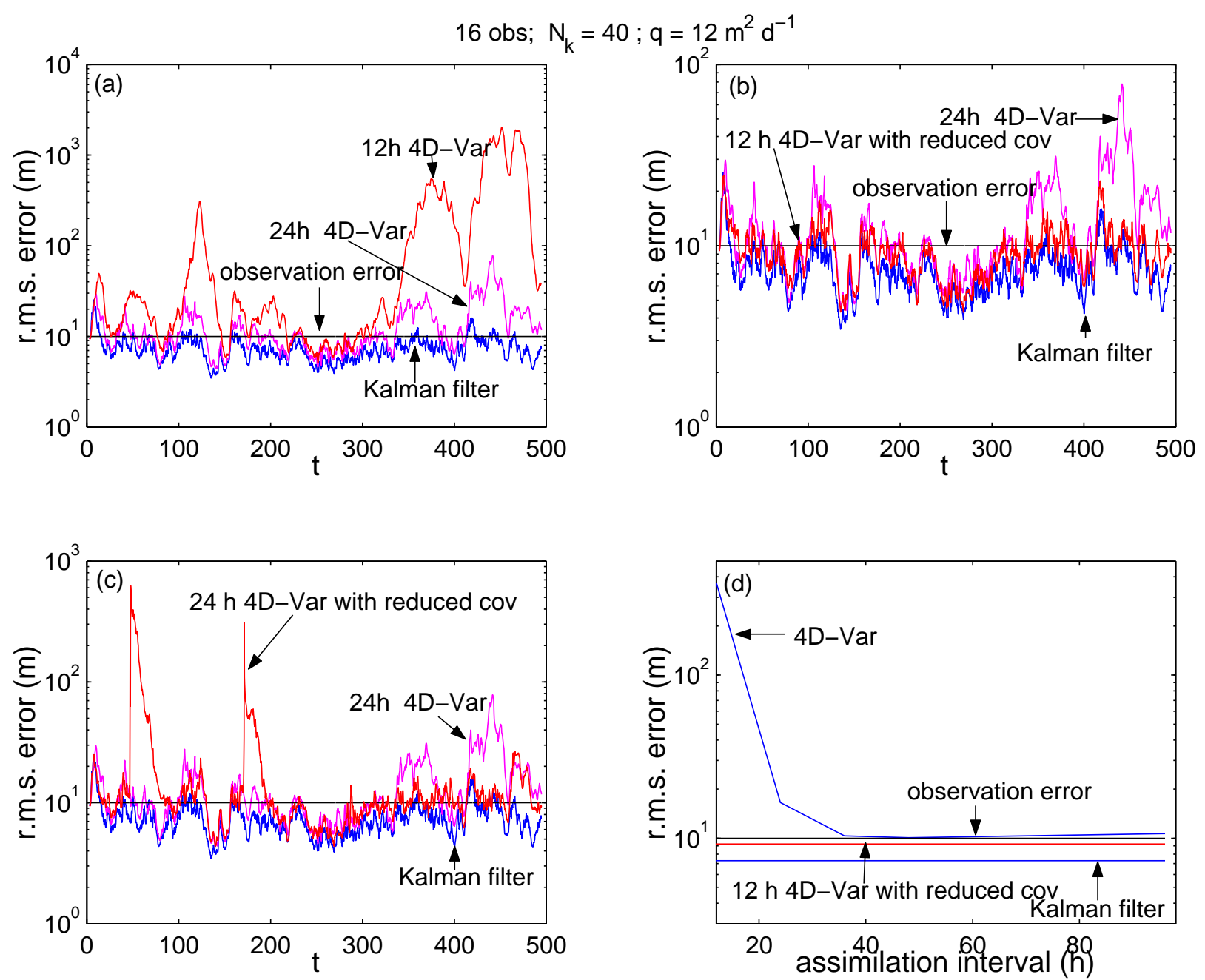

Figure 14: Error in a simulation of the time dependent storm track model with model error. Panel (a): comparison of the errors in $a 12 \mathrm{~h}$ and $24 \mathrm{~h} 4 \mathrm{D}$-Var with the error in the full Kalman filter. Panel $(b)$ : comparison of the error in a 24 h 4D-Var with the error in a 12 h 4D-Var in which the isotropic static $\mathbf{B}$ has been preconditioned with the error covariance obtained from a reduced rank Kalman filter with balanced truncation. The reduced rank Kalman filter has been obtained with model error. In the truncated system 40 dof have been retained out of the 400 dof of the system. The isotropic $\mathbf{B}$ introduced to the reduced rank covariance has amplitude equal to the smallest eigenvalue of the reduced rank covariance. Also shown is the error resulting from the Kalman filter. The $12 \mathrm{~h} 4 \mathrm{D}$-Var performance is nearly optimal. Panel (c): comparison of the error in a 24 h 4D-Var with the error in a $24 h$ 4D-Var in which the isotropic static $\mathbf{B}$ has been preconditioned with the error covariance obtained from the reduced Kalman filter. The $24 \mathrm{~h} 4 \mathrm{D}$-Var preconditioned with the covariance from the reduced Kalman filter propagates the covariance without model error longer and its performance is worse than that of the corresponding 12 h 4D-Var. Panel (d): r.m.s. error in 4D-Var assimilations in the time dependent storm track model with model error as a function of assimilation interval. Also shown is the error obtained with sequential application of a Kalman filter and the error from the $12 \mathrm{~h}$ 4D-Var which was preconditioned with the reduced rank covariance. 16 observations are assimilated with r.m.s. observational error of $10 \mathrm{~m}$. The model error variance coefficient is $q=12 \mathrm{~m}^{2} \mathrm{~d}^{-1}$, so that a model error of $5 \mathrm{~m}$ accumulates after a day. 


\section{Conclusions}

A data assimilation system combines observations and dynamics expressed through a numerical forecast model to obtain an estimate of the state of the atmosphere. An optimal data assimilation system combines observations and dynamics to obtain the statistically best state estimate. Statistical optimality requires information about the observation error and about the error in the numerical forecast. This latter is difficult to obtain because of the high dimension of the error system so that approximations to the forecast error have to be made to implement practical applications of optimal state estimation. A promising method for obtaining an approximation to forecast error is to advance the error covariance in a state space of reduced dimension compared with that of the full forecast error system. The error covariance in the reduced space can then be used in an approximate optimal state estimation method such as 4D-Var or the extended Kalman filter. Such a reduction is possible because the significantly unstable subspace of the error system is of much lower dimension than the complete state dimension.

Assimilation systems can be usefully modelled as observer systems in which any gain matrix that stabilizes the analysis error system is an observer and the gain that results in minimum analysis error is the optimal observer. This perspective on assimilation provides insight by allowing generalized stability analysis of the observer system to be performed revealing for instance the distributed error sources that serve to most effectively degrade the analysis (Farrell and Ioannou, 2003).

Analysis of the observer system modelling 4D-Var and the Kalman filter reveals that as the number of observations assimilated increases the analysis error asymptotes to a finite value comparable to observational error and independent of the number of observations unless the forecast error covariance is systematically adjusted to account for the increase of observations. One way this adjustment can be accomplished is by advancing the forecast error covariance in the dynamically relevant reduced order system that supports the growing error structures.

The result from using this accurate forecast covariance is that as the number of observations $n$ increases the associated Kalman filter obtains assimilation error $O\left(n^{-1 / 4}\right)$ (with model error present) while the 4D-Var simulation fails to systematically reduce the estimation error. Assuming that redundancy of observation in the restricted subspace of significantly growing error structures has or soon will be available it is important to systematize the error covariance calculation in order to take advantage of these observations.

The gain under the assumption of a perfect model develops far field loading that degrade the assimilation because the model error is in fact non-vanishing. The error covariance obtained by introducing model error into the reduced system suppresses these far field loadings. The error covariance calculated in the reduced system provides a method for introducing model error into 4D-Var thus reducing the deleterious effects of the perfect model assumption and allowing accurate equivalent gains to be realized on short assimilation intervals.

\section{Acknowledgements}

This work was supported by NSF ATM-0123389 and by ONR N00014-99-1-0018.

\section{References}

Bishop, C. H., B. J. Etherton, S. J. Majumdar, and J. Sharanya, 2001: Adaptive sampling with the ensemble transform Kalman filter. Part I: Theoretical aspects. Mon. Wea. Rev., 129, 420-436.

Buizza, R, and T. Palmer, 1995: The singular vector structure of the atmospheric general circulation.J. Atmos. 
Sci. 52, 1434-1456.

Cohn, S. E. and R. Todling, 1966: Approximate Kalman filters for stable and unstable dynamics. J. Meteor. Soc. Japan, 75, 257-288.

Dee, D. P., 1995: On-line estimation of error covariance parameters for atmospheric data assimilation. Mon. Wea. Rev., 123,1128-1145.

Delsole, T. M. and B. F. Farrell, 1994: Nonlinear Equilibration of Localized Instabilities on a Baroclinic Jet. J. Atmos. Sci. , 51, 22702284.

Evensen, G., 1994: Sequential data assimilation with a nonlinear quasi-geostrophic model using Monte-Carlo methods to forecast error statistics. J. Geophys. Res., 99 (C5), 10143-10162.

Farrell, B. F. 1982: Pulse Asymptotics of the Charney Baroclinic Instability Problem. J. Atmos. Sci. , 39, 507517.

Farrell, B. F., and P. J. Ioannou, 1996: Generalized Stability. Part I: Autonomous Operators. J. Atmos. Sci. , 53, 2025-2041.

Farrell, B. F., and P. J. Ioannou, 1999: Perturbation growth and structure in time dependent flows. J. Atmos. Sci. , 56, 3622-3639.

Farrell, B. F., and P. J. Ioannou, 2001a: Accurate Low Dimensional Approximation of the Linear Dynamics of Fluid Flow. J. Atmos. Sci. , 5827712789.

Farrell, B. F., P. J. Ioannou, 2001b: State Estimation Using a Reduced-Order Kalman Filter. J. Atmos. Sci. , 58, 36663680.

Farrell, B. F., and P. J. Ioannou, 2003: Optimal excitation of linear dynamical systems by distributed forcing. J. Atmos. Sci. (submitted).

Fukumori, I., and P. Malanotte-Rizzoli, 1995: An approximate Kalman filter for ocean data assimilation; an example with an idealized Gulf Stream model. J. Geophys. Res. Oceans, 100, 6777-6793.

Gelaro, R., C. Reynolds, and R. M. Errico, 2000: Transient and asymptotic perturbation growth in a simple model. 128, Quart. J. Roy. Meteor. Soc. , 205-228.

Ghil, M., 1997: Advances in sequential estimation for atmospheric and oceanic flows. J. Meteor. Soc. Japan, 75, 289-304.

Ghil, M., and P. Malanotte-Rizzoli, 1991: Data assimilation in meteorology and oceanography. Adv. in Geophys., 33, 141-266.

Ghil, M., and R. Todling, 1996: Tracking atmospheric instabilities with the Kalman filter. Part II: Two layer results. Mon. Wea. Rev., 124, 2340-2352.

Glover, K., 1984: An optimal Hankel-norm approximation of linear multivariable systems and their $\mathbf{L}^{\infty}$-error bounds. Int. J. Control, 39, 1115-1193.

Houtekamer, P. L., and H. L. Mitchell, 1998: Data assimilation using an ensemble Kalman filter technique, Mon. Wea. Rev., 126, 796-811.

Ide, K., and M. Ghil, 1997: Extended Kalman filtering for vortex systems. Part I: Methodology and point vortices. Dyn. Atmos. Oceans,27, 301332.

Ide, K., P. Courtier, M. Ghil, M., and A. C. Lorenc, 1997: Unified notation for data assimilation: operational, sequential, and variational. J. Meteor. Soc. Japan, 75,181-189.

Illyashenko, Yu. S., 1983: On the dimension of attractors of k-contracting systems in infinite-dimensional space. Vestn. Mosk. Univ. Ser. 1 Mat. Mekh., 3, 52-58.

Kalman, R.E., 1960: A new approach to linear filtering and prediction problems. J. Basic Eng.. 82D, 35-45.

Kaplan, J. L., and J. A. Yorke, 1979: Preturbulence: a regime observed in a fluid flow model of Lorenz. Comm. Math. Phys., 67, 93-108.

Lermusiaux, P.F.J., and A. R. Robinson, 1999: Data assimilation via error statistical estimation. Part I: Theory and schemes. Mon. Wea. Rev., 127, 1385-1407.

Lin, S.J., and R. T. Pierrehumbert, 1993: Is the Midlatitude Zonal Flow Absolutely Unstable?. J. Atmos. Sci. , 50, 505517.

Miller, R. N., M. Ghil, and F. Gauthiez, 1994: Advanced data assimilation in strongly nonlinear dynamical 
systems.J. Atmos. Sci.,51, 10371056.

Moore, B. C., 1981: Principal component analysis in linear systems: controllability, observability, and model reduction. IEEE Trans. on Automatic Control, AC-26, 17-31.

North, G., 1984: Empirical orthogonal functions and normal modes. J. Atmos. Sci. , 41, 879-887.

Palmer, T. N., R. Gelaro, J. Barkmeijer, and R. Buizza, 1998: Singular vectors, metrics, and adaptive observations. J. Atmos. Sci. , 55, 633-653.

Reynolds, C. A., and R. M. Errico, 1999: Convergence of singular vectors towards Lyapunov vectors. Mon. Wea. Rev., 127, 2309-2323.

Stewart, G. W., and J-G. Sun, 1990: Matrix Perturbation Theory, Academic Press, Boston, Massachusetts, pp. 365.

Sznaier, M., A. C. Doherty, M. Barahona, J. C. Doyle, and H. Mabuchi, 2002: A new bound of the $L_{2}([0, T])$ induced norm and applications to model reduction. Proc. 2002 American Control Conference.

Takens, F., 1981: in Dynamical Systems and Turbulence, Lecture Notes in Mathematics Vol. 898, edited by D. Rand and L.-L. Young (Springer-Verlag, Berlin,1981), pp. 366-381.

Tippett, M. K., S. E. Cohn, R. Todling, and D. Marchesin, 2000: Low-dimensional representation of error covariance. Tellus, 52,533-553.

Todling, R., and M. Ghil, 1994: Tracking atmospheric instabilities with the Kalman filter. Part I: Methodology and one-layer results. Mon. Wea. Rev., 122, 183-204.

Zhou, K., and J. C. Doyle, 1998: Essentials of Robust Control, Prentice Hall, Upper Saddle River, New Jersey, pp 411.

Van Dooren, P., 2000: Gramian based model reduction of large-scale dynamical systems, in Numerical Analysis 1999, Chapman and Hall, CRC Press, London, pp. 231-247.

Verlaan, M. and A. W. Heemink, 1997: Tidal flow forecasting using reduced rank square root filters. Stochastic Hydrology and Hydraulics, 11, 349-368.

Wunsch, C., 1996: The Ocean Circulation Inverse Problem, Cambridge University, Cambridge, pp. 442. 